\title{
Virulence determinants of pandemic influenza viruses
}

\author{
Donna M. Tscherne ${ }^{1}$ and Adolfo García-Sastre ${ }^{1,2,3}$
}

1Department of Microbiology, ${ }^{2}$ Department of Medicine, Division of Infectious Diseases, and ${ }^{3}$ Global Health and Emerging Pathogens Institute, Mount Sinai School of Medicine, New York, New York, USA.

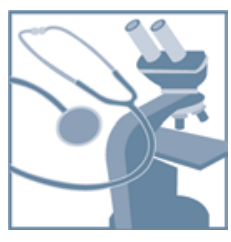

Influenza A viruses cause recurrent, seasonal epidemics and occasional global pandemics with devastating levels of morbidity and mortality. The ability of influenza A viruses to adapt to various hosts and undergo reassortment events ensures constant generation of new strains with unpredictable degrees of pathogenicity, transmissibility, and pandemic potential. Currently, the combination of factors that drives the emergence of pandemic influenza is unclear, making it impossible to foresee the details of a future outbreak. Identification and characterization of influenza A virus virulence determinants may provide insight into genotypic signatures of pathogenicity as well as a more thorough understanding of the factors that give rise to pandemics.

\section{Influenza A virus biology}

Influenza A virus, a member of the family Orthomyxoviridae, consists of 8 single-stranded, negative-sense viral RNA (vRNA) segments and encodes up to 11 proteins (Figure 1 and ref. 1 ). The genome is encapsidated in a lipid bilayer that is studded with the hemagglutinin (HA) and neuraminidase (NA) glycoproteins and traversed by the matrix protein 2 (M2) proton channel. Viruses are divided into subtypes based on genetic and antigenic differences in their HA and NA surface glycoproteins. To date, there are $16 \mathrm{HA}$ (H1-H16) and 9 NA (N1-N9) influenza A subtypes, all of which are circulating in avian species (2). The natural hosts of influenza $A$ viruses are wild waterfowl and shorebirds, although these viruses have adapted to many avian and mammalian species (2).

To initiate infection, influenza virus HA binds to sialic acid-containing receptors on the target cell surface, triggering receptormediated endocytosis and subsequent fusion within the low-pH environment of the endosome (1). Oligomerized M2 protein forms an ion channel necessary for viral uncoating. Inside the virion, each influenza vRNA segment is coated with nucleoprotein (NP) to form a ribonucleoprotein (RNP) and is associated with the RNAdependent RNA polymerase complex of three proteins, PB1, PB2, and PA. Each RNP interacts with matrix protein 1 (M1), which lies beneath the lipid bilayer. Within the nucleus of an infected cell, the viral genome serves as a template for synthesis of mRNA and complementary RNA (cRNA), the latter of which functions as a template for genomic RNA (i.e., vRNA) replication. Assembly and budding of virions occurs at the cell surface in lipid raft microdomains (3). The NA protein cleaves sialic acid moieties from sialyloligosaccharides and facilitates the release of nascent virions. The nonstructural (NS) gene encodes two proteins: NS1 functions in evading host immune responses by limiting cellular IFN production and the activity of IFN-stimulated genes (ISGs); NEP mediates the export of viral RNPs (vRNPs) from the nucleus. PB1-F2 is a small protein, encoded from an alternative reading frame of the PB1 gene segment, that is thought to play a role in viral patho-

Conflict of interest: A. García-Sastre reports a financial interest with Vivaldi BioScience and is also involved with patents filed through Mount Sinai School of Medicine related to plasmid-based rescue of influenza virus and antiviral targets for influenza. Citation for this article: J Clin Invest. 2011;121(1):6-13. doi:10.1172/JCI44947. genicity (4-7). Recently, reverse genetic systems for generation of influenza A viruses from cloned cDNAs have been established and used to analyze influenza biology and pathogenesis $(8,9)$.

Influenza virulence and pathogenicity can be measured using parameters of morbidity and mortality within animal models. Influenza-infected mice experience a decline in body weight correlating with severity of disease, although the standard disease symptoms of human influenza, such as fever and respiratory discharge, are not observed (10). Ferrets, however, develop many of the complex clinical manifestations of influenza-infected humans (11-13), including viral replication in the upper and lower respiratory tracts and the ability to transmit virus via contact and aerosol routes (14). For transmission studies, researchers have also used guinea pigs, which are more amenable to the laboratory setting (15-19). Pigs can be experimentally infected with influenza viruses and develop clinical symptoms (20). Nonhuman primates, such as macaques, also serve as an appropriate model for human influenza disease and may additionally be used for evaluation of innate and adaptive immune responses (21).

\section{Influenza A virus pandemics}

The complex interplay of factors that drive the emergence of pandemic influenza is poorly understood. An influenza virus pandemic is normally initiated when a virus that transmits from human to human acquires a new HA molecule to which a significant percentage of the human population is immunologically naive (22). This can occur either when an avian virus reassorts with a human virus during coinfection of an infected mammal or when an animal virus with a novel HA adapts for efficient human-to-human transmission and can cause significant illness (22). Three times in the previous century, in 1918, 1957, and 1968, influenza pandemics have emerged in the human population, with devastating consequences (23). Moreover, the recent swine-origin influenza virus (S-OIV) H1N1 pandemic of 2009 and the emerging H5N1 highly pathogenic avian influenza (HPAI) virus further emphasize the looming risk that influenza viruses pose to humans.

1918 Spanish influenza (H1N1). The Spanish influenza pandemic of 1918-1919 was the most devastating in documented history, infecting hundreds of millions and claiming more than 20 million lives worldwide (24). The 1918 pandemic was unusual in its 


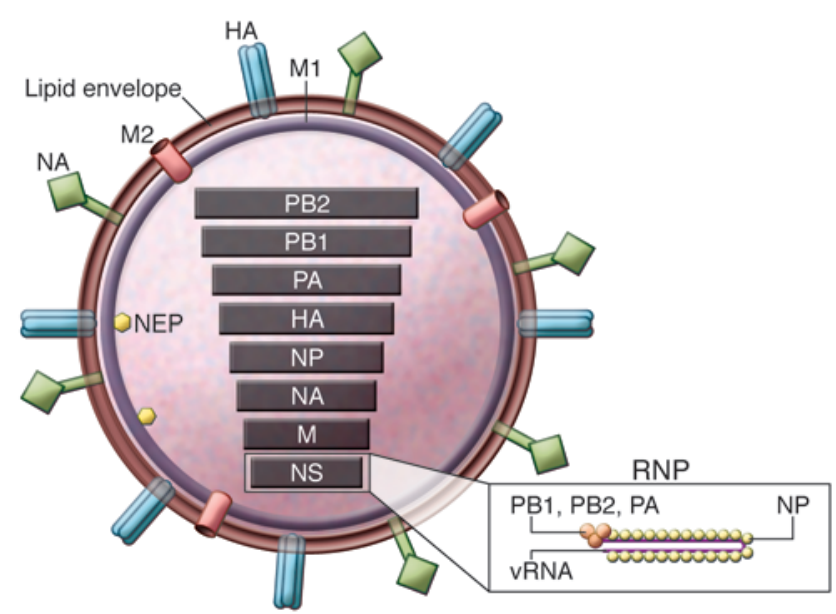

disproportionately high mortality rate in young adults aged 15-34 years (22). Analysis of preserved human lung tissues from fatally infected patients revealed a rapid destruction of the respiratory epithelium and profound, severe lung pathology (25). The most common cause of death from complications of 1918 influenza virus was secondary bacterial pneumonia (26), but viral pneumonia also developed in many patients.

Recently, the genome of the 1918 pandemic influenza virus was completely sequenced $(27,28)$, and the virus was reconstructed using reverse genetics (29). Consistent with its high morbidity and mortality phenotype in humans, reconstructed 1918 influenza virus caused death in embryonated chicken eggs, mice (29), ferrets (30), and macaques (31). However, this virus was not lethal for pigs, chickens, or ducks (32). The high virulence phenotype of the 1918 pandemic virus has been mapped to HA and NA, the replication complex, NS1, and PB1-F2 proteins, although the full constellation of 1918 genes is essential for optimal virulence in mice (29, 31). Efficient transmission of 1918 virus between ferrets was shown to require HA and PB2 (33). Gene expression profiling of infected mice and macaques revealed enhanced, global activation of host genes involved in inflammation and cell death responses $(31,34)$.

1957 Asian (H2N2) and 1968 Hong Kong (H3N2) influenza viruses. In the winter of 1957 in southern China, a pandemic influenza virus bearing an H2 HA and an N2 NA of avian virus origin emerged in the human population (35). This virus also harbored an avianderived PB1 segment (36). By 1958, the H2N2 Asian influenza virus had replaced the previously circulating H1N1 descendents of the 1918 virus, and the death toll in the United States had reached approximately 70,000 people (37). These H2N2 viruses circulated in the human population until 1968, when they were replaced by another human/avian reassortant virus, first isolated in Hong Kong. This virus retained the N2 NA from the circulating H2N2 viruses, but acquired an $\mathrm{H} 3 \mathrm{HA}$, as well as a PB1 segment, of avian origin $(35,36)$. By 1970 , nearly 34,000 people in the United States had died from Hong Kong pandemic influenza (37).

1977 Russian influenza (H1N1). First reported in China in the spring of 1977, an H1N1 virus began to cause disease in young adults under the age of 23 years. The high level of similarity between this virus and those circulating in the early 1950s prior to the H2N2 outbreak (38) prompts the suspicion that the 1977 $\mathrm{H} 1 \mathrm{~N} 1$ virus was an accidental release from a laboratory stock. This assumption is also consistent with the virus being pathogenic in

\section{Figure 1}

Influenza A virus particle. Three integral membrane proteins, HA, NA, and the M2 ion channel, are inserted into the viral lipid envelope. The M1 matrix protein lies beneath the envelope. The viral genome is composed of eight segments of VRNA, coated with NP to form VRNPs. The viral transcriptase complex, containing PB1, PB2, and PA, is associated with each RNP. Small amounts of NEP, encoded from the NS gene, are found in purified virions. the younger demographic; older adults already had substantial immunity to these viruses. After 1977, the reemerged H1N1 virus began to cocirculate with the $\mathrm{H} 3 \mathrm{~N} 2$ viruses that were present at the time and continues to do so today (39). Notably, there has been reassortment between the two cocirculating strains, generating an H1N2 virus first isolated in the 2001-2002 influenza season (40). These H1N2 viruses failed to cause appreciable disease and did not persist in subsequent influenza seasons (41).

2009 S-OIV (H1N1). During March and April of 2009, several areas in Mexico began reporting large numbers of patients presenting with influenza-like illness (42). By June 11, 2009, the causative agent, 2009 S-OIV, had spread to 74 countries and been confirmed in nearly 29,000 patients, prompting the World Health Organization to raise the global pandemic alert level to 6 , the pandemic phase (43). As of late July 2010, cases of pandemic 2009 S-OIV were confirmed in 214 countries and resulted in more than 18,000 deaths (43).

Genetic analysis suggests that 2009 S-OIV is composed of segments from two H1N1 swine virus lineages: classical swine viruses, which are descendents of the 1918 human influenza virus, and European avian-like influenza viruses (Figure 2 and refs. 44, 45). In the late 1990s, reassortment among North American avian, human $\mathrm{H} 3 \mathrm{~N} 2$, and classical swine H1N1 viruses resulted in triple-reassortant $\mathrm{H} 3 \mathrm{~N} 2$ and $\mathrm{H} 1 \mathrm{~N} 2$ swine viruses. A swine triple-reassortant virus then reassorted with a Eurasian avian-like swine virus, resulting in 2009 S-OIV. The PB2 and PA segments were of avian virus lineage, whereas PB1 was contributed by the human seasonal $\mathrm{H} 3 \mathrm{~N} 2$ virus $(44,45)$. The HA, NP, and NS genes are of classical swine virus origin. The remaining segments, NA and $M$, are from the European avian-like H1N1 lineage $(44,45)$. The ancestor of the 2009 S-OIV pandemic virus appears to have been circulating undetected in an animal reservoir, most likely pigs, for some time prior to the 2009 human outbreak (46).

Clinically, infection with pandemic 2009 S-OIV appears to be indistinguishable in severity and symptoms from seasonal H1N1 or H3N2 influenza, with some notable exceptions $(47,48)$. First, a greater percentage of patients have complained of gastrointestinal symptoms than what is usually reported for seasonal influenza (48). Correspondingly, viral replication of S-OIV within the intestinal tracts of inoculated ferrets has been documented $(49,50)$. A second striking feature is the high rate of infection and severe disease in the younger population, rather than in the elderly, as for seasonal influenza. A putative explanation for this observation is 


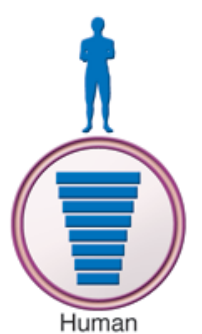

(H3N2)
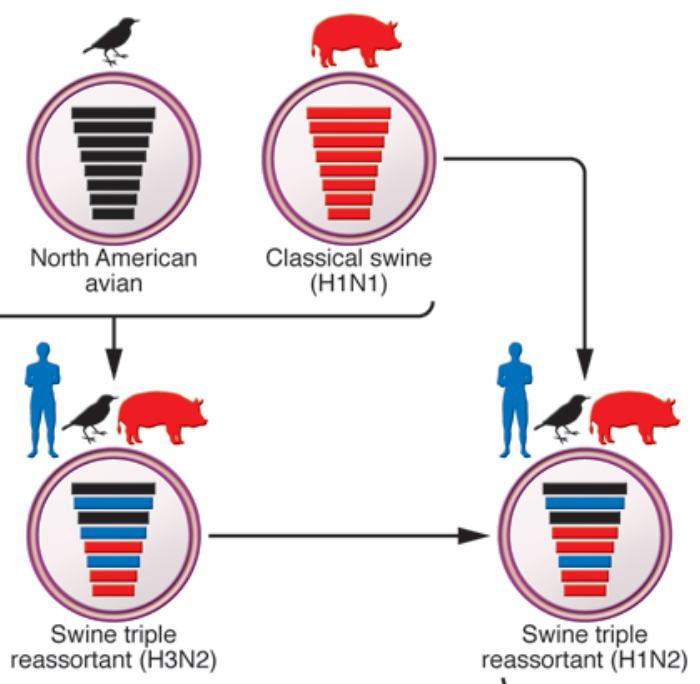
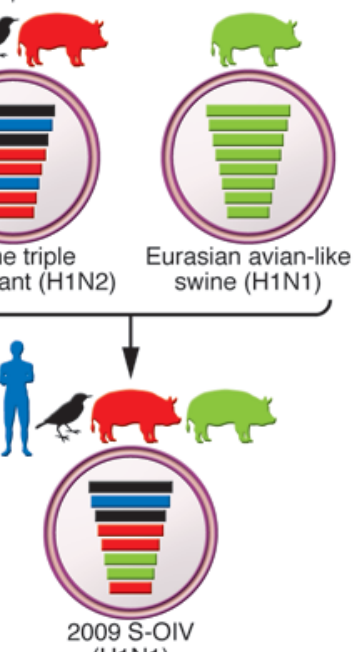

(H1N1)

\section{Figure 2}

Origin of 2009 S-OIV. Reassortment of North American swine $\mathrm{H} 3 \mathrm{~N} 2$ and $\mathrm{H} 1 \mathrm{~N} 2$ triple-reassortant viruses (of North American avian, human [H3N2], and classical swine [H1N1] origin) with Eurasian avian-like swine viruses (H1N1) resulted in the 2009 S-OIV pandemic virus. Each gene segment of avian, human, or swine origin corresponds to a characteristic feature on the surface of the schematic viral particle. the presence of cross-reactive antibodies generated during previous exposures to 1918-like viruses, which circulated between 1918 and 1943 , or H1N1 influenza vaccination in 1976, both of which were shown to confer protection to 2009 S-OIV in mice (51). Structural data demonstrate that 2009 S-OIV lacks particular glycosylation sites in the HA head region that have been gradually acquired by human H1 HAs to mask neutralizing epitopes $(52,53)$, making 2009 S-OIV antigenically similar to viruses that circulated after the 1918 pandemic. These data suggest that HAs in swine viruses can remain antigenically frozen in time because of the lack of selection pressure in this host and can reemerge in the future.

The 2009 S-OIV virus contains none of the hallmarks of highly pathogenic influenza viruses (see below), although results from infection of animal models suggest that this virus produces a more severe disease than seasonal H1N1 viruses $(49,50,54,55)$. In mice, ferrets, and nonhuman primates, 2009 S-OIV replicated efficiently in the upper and lower respiratory tracts and induced more pronounced lung pathology than seasonal H1N1 viruses $(49,50,54$, 55). Induction of proinflammatory cytokines was also markedly increased in the lungs of mice and macaques following 2009 S-OIV infection compared with seasonal H1N1 virus infection (54). Aerosol transmission between ferrets was comparable to $(50,54,55)$, or slightly less efficient than (49), seasonal H1N1 viruses. Miniature pigs displayed few disease symptoms after infection with 2009 S-OIV, despite high levels of viral replication in the lungs (54). This observation agrees with the hypothesis that 2009 S-OIV had been circulating within the swine population asymptomatically prior to its transmission to humans, although definitive evidence will require the isolation of recent ancestors of 2009 S-OIV $(46,54)$.
Overall, the results from experiments in these animal models suggest that the 2009 S-OIV virus is more pathogenic than seasonal H1N1 strains. However, pathogen-free animal models may be more reflective of the disease observed in the immunologically naive, younger human population, which was more susceptible to severe disease. Moreover, all seasonal, contemporary H1N1 strains are not created equally. A larger panel of selected $\mathrm{H} 1 \mathrm{~N} 1$ viruses, which vary in their replication, virulence, and transmissibility, may provide a more appropriate comparison to evaluate the pathogenic potential of the 2009 S-OIV virus.

Emerging H5N1 HPAI viruses. Since 1997, H5N1 HPAI viruses have spread throughout Asia into Europe and Africa, causing outbreaks in poultry populations and an increasing number of human zoonotic infections and fatalities $(56,57)$. H5N1 HPAI viruses can cause a fatal infection in chickens, as well as waterfowl, regarded as the natural reservoir of influenza A viruses. Many are also lethal in a variety of mammalian species, such as cats, ferrets, and mice. In humans, H5N1 HPAI viruses appear to induce a more systemic disease than human influenza; in addition to high viral titers in the respiratory tract, viral antigen or RNA is detectable in blood, cerebrospinal fluid, and internal organs (58-60). Patients with H5N1 HPAI often progress to severe pneumonia, followed by acute respiratory distress syndrome, resulting in death in approximately $60 \%$ of cases $(58,61)$. Currently, H5N1 HPAI transmits very poorly between humans, although some instances have been reported (62). Nevertheless, there is concern that $\mathrm{H} 5 \mathrm{~N} 1$ will acquire the ability to spread person to person, either by accumulating fortuitous mutations or by reassortment with a human strain (23). 


\section{Table 1}

Influenza proteins and pathogenicity determinants

\begin{tabular}{|c|c|c|c|c|c|}
\hline \multirow[t]{2}{*}{ Protein } & \multirow[t]{2}{*}{ Segment } & \multirow[t]{2}{*}{ Function } & \multicolumn{2}{|c|}{ Pathogenicity determinant } & \multirow[t]{2}{*}{ Reference } \\
\hline & & & High & Low & \\
\hline PB2 & 1 & Polymerase cofactor, binds most mRNA caps & $627 \mathrm{~K}^{\mathrm{A}}$ & $627 \mathrm{E}^{\mathrm{B}}$ & 80 \\
\hline PB2 & 1 & Polymerase cofactor, binds most mRNA caps & $701 \mathrm{~N}$ & 7010 & 89 \\
\hline PB1 & 2 & Polymerase cofactor, RNA-dependent RNA polymerase & - & - & - \\
\hline PB1-F2 & 2 & Proapoptotic & $66 \mathrm{~S}$ & $66 \mathrm{~N}$ & 5 \\
\hline PA & 3 & Polymerase cofactor endonuclease activity, elongation factor? & - & - & - \\
\hline $\mathrm{HA}$ & 4 & Membrane glycoprotein, binding, and fusogenic functions & Multi-basic cleavage site & Single-basic cleavage site & 79 \\
\hline NP & 5 & Component of RNP, encapsidates vRNA segments & - & - & - \\
\hline NA & 6 & Membrane glycoprotein, sialidase & $274 Y c$ & $274 \mathrm{H}$ & - \\
\hline M1 & 7 & Lies under the viral envelope & - & - & - \\
\hline M2 & 7 & Membrane protein, forms ion channel & - & - & \\
\hline NS1 & 8 & Evasion of host immune response & $92 \mathrm{E}$ & $92 \mathrm{D}$ & 102 \\
\hline NS1 & 8 & Evasion of host immune response & C-terminal E-S-E-V motif & C-terminal deletion & 105,106 \\
\hline NEP & 8 & Nuclear export of vRNPs & - & - & - \\
\hline
\end{tabular}

APathogenic with respect to mammalian, not avian, hosts. ${ }^{\mathrm{B} C a n}$ be compensated by other PB2 changes. ${ }^{\mathrm{C}}$ Second-site mutations counteract the loss in fitness from the NA H274Y oseltamivir resistance mutation.

\section{Role of influenza A genes in pathogenicity and virulence}

The pathogenicity of influenza virus is considered multigenic; it is determined by the constellation of genes within a particular influenza virus strain within a specific host. There are, however, particular genetic mutations that can enhance various aspects of the viral life cycle, including virus binding and entry, genome transcription and translation, virion assembly and release, and evasion of innate immune responses (Table 1). These so-called virulence determinants can contribute to a highly pathogenic phenotype in infected animals.

$H A$. The HA protein performs the binding and fusogenic functions for influenza A virus and thereby controls a pivotal point in virus infection. HA plays a critical role in adaptation to certain hosts through its affinity for receptors differentially expressed between species. Human influenza viruses have a preference for receptors terminating in $\alpha 2,6$-linked sialic acid moieties, which are present on the epithelium of the human respiratory tract, whereas avian influenza viruses prefer $\alpha 2,3$-linked sialic acids, found predominantly in the avian intestinal epithelium (Figure $3 \mathrm{~A}$ and refs. 63-66). The specific residues within HA that control the receptor specificity of human and avian viruses have been determined and differ by subtype. H1 HAs with human-like receptor binding properties contain D190 and D225 (H3 numbering), whereas avian H1 HAs have E190 and G225. For H2 and H3 HAs, receptor binding is dictated by L226 and S228 for human virus and Q226 and G228 for avian virus (Figure 3B and ref. 67).

The adaptation of an avian influenza A virus to recognize humantype receptors is considered a requirement for efficient human-tohuman transmission and thus for the development of a pandemic virus (65). The pandemic viruses of 1918, 1957, and 1968 had HAs of avian origin, but acquired the ability to bind human-like receptors $(65,68)$. Likewise, only a 1918 virus with human-type receptor preference could transmit efficiently between ferrets; mixed specificity for avian and human receptors reduced transmission in the ferret model (30). Numerous amino acid changes that confer increased affinity for human-like receptors have been identified in H5N1 HPAI viruses (69-73). However, to date, none of these isolates are able to bind to human $\alpha 2,6$-linked sialic acid receptors exclu- sively. These studies suggest that receptor specificity contributes to the lack of sustained transmission of H5N1 between humans.

HA receptor preference appears to affect transmission by controlling the anatomical site of viral replication (74). Both $\alpha 2,6$ - and $\alpha 2,3$ linked sialic acids can be found on cells within the respiratory tract of humans, but in different locations. $\alpha 2,6$-linked sialic acids are preferentially expressed on cells in the human upper respiratory tract, while $\alpha 2,3$-linked receptors are found on cells deeper in the lungs. Replication in the upper airway may be required for efficient transmission and initiation of a productive infection in humans (74). The presence of avian-type receptors in human lungs may also explain the severe pneumonia in human cases of $\mathrm{H} 5 \mathrm{~N} 1$ infection, indicative of viral replication in the lower respiratory tract $(74,75)$. Moreover, the presence of $\alpha 2,6$ - and $\alpha 2,3$-linked sialic acid receptors on swine tracheal epithelial cells allows transmission of both avian and human viruses to pigs (63). This supports the hypothesis that these animals can serve as a mixing vessel for the reassortment of novel influenza A viruses and their subsequent transmission to humans.

A major virulence determinant in influenza virus is a multi-basic cleavage site within HA. Cleavage of the HA precursor, HA0, into HA1 and HA2 exposes the amino terminus of HA2 containing the fusion peptide, allowing for virus envelope fusion with a host endosomal membrane. Cleavage susceptibility of HA0 correlates well with the pathogenicity of HPAI viruses in poultry (76-78). In low pathogenic avian influenza (LPAI) viruses, cleavage of HA at a conserved arginine is mediated by host trypsin-like enzymes, thereby restricting infection to tissues in which these enzymes are present. In contrast, the HA0 cleavage sites of H5 and H7 HPAI viruses are a multi-basic stretch of amino acids, which can be recognized by ubiquitous host enzymes, such as furin and PC6, allowing systemic infection (79). In mice, the multi-basic cleavage site was required for $\mathrm{H} 5 \mathrm{~N} 1$ virulence and viral spread to the mouse brain following intranasal infection (80). Influenza viruses containing a multi-basic cleavage site in the HA have only been found among H5 and H7 subtypes, and this trait appears to be selected only during poultry infections, most likely because it facilitates rapid spread in poultry. To date, no human influenza virus with a multi-basic cleavage site in the HA has been isolated from nature. 
A
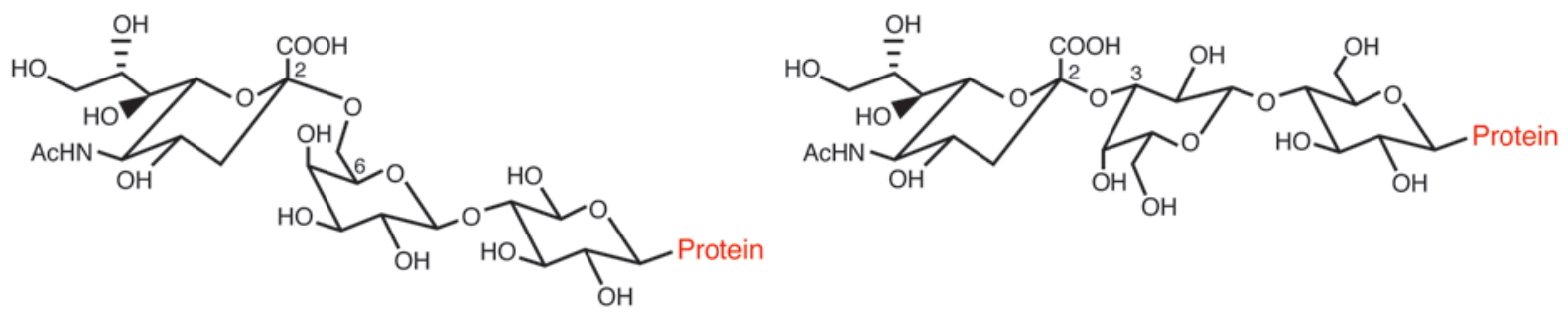

B

\begin{tabular}{|c|c|c|}
\hline Subtype & Human preference & Avian preference \\
\hline $\mathrm{H} 1$ & D190, D225 & E190, G225 \\
\hline $\mathrm{H} 2 / \mathrm{H} 3$ & L226, S228 & Q226, G228 \\
\hline
\end{tabular}

Figure 3

Avian versus human influenza virus preference for sialic acid receptor linkages. (A) Sialic acid $\alpha 2,6$ and $\alpha 2,3$ linkage to a cell surface glycoprotein is bound preferentially by HA of human and avian influenza viruses, respectively. (B) Crucial residues in HA that dictate receptor preference for human or avian influenza viruses.

PB2. The viral polymerase complex has been recognized as an important contributor to viral pathogenicity, most likely by directly controlling levels of vRNA replication. Numerous substitutions within the PB2 subunit have been shown to alter host range and virulence. A glutamic acid at PB2 position 627 is generally found in avian viruses, whereas nearly all human isolates carry a lysine at this position. H5N1 viruses with a PB2 E627K mutation cause a lethal, systemic infection in mice, but become nonpathogenic for mammals if this residue remains a glutamic acid (80). Moreover, avian viruses quickly acquire the $\mathrm{E} 627 \mathrm{~K}$ change after a single passage in mice $(81,82)$, and many of the $\mathrm{H} 5 \mathrm{~N} 1$ viruses isolated from human hosts carry a PB2 with $\operatorname{E627K}(59,83,84)$.

Exactly how PB2 627K enhances pathogenicity and adaptation to mammalian hosts has been the subject of much investigation. Available data suggest that $\mathrm{PB} 2$ position 627 determines the temperature sensitivity of vRNA replication (85). Human influenza viruses replicate in the upper respiratory tract at an approximate temperature of $33^{\circ} \mathrm{C}$, whereas avian influenza viruses prefer the intestinal tract, at a temperature of nearly $41^{\circ} \mathrm{C}$. Viruses with PB2 $627 \mathrm{~K}$ can efficiently replicate in the mammalian upper respiratory tract, whereas those that possess PB2 627E cannot (81). A PB2 E627K mutation enhances avian virus replication in mammalian cells at $33^{\circ} \mathrm{C}$, but not $37^{\circ} \mathrm{C}$ or $41^{\circ} \mathrm{C}$, in vitro (85). In addition, $\mathrm{H} 5 \mathrm{~N} 1$ viruses with a PB2 $\mathrm{E} 627 \mathrm{~K}$ mutation have increased growth in the nasal passages of mice compared with the wild-type virus (81). The cold sensitivity of avian virus polymerases with PB2 627E may be the result of a disrupted interaction between PB2 and NP (86), the presence of a cellular restriction factor (87), or, conversely, the lack of a compatible host cofactor for polymerase activity (88). The amino acid at PB2 627 also affects the transmissibility of influenza viruses. A PB2 K627E mutation reduces transmission of human influenza viruses in the guinea pig, presumably because of reduced replicative ability in the upper respiratory tract (18).

A PB2 D701N mutation is also associated with increased influenza virus virulence in mammals. Having PB2 $701 \mathrm{~N}$ increases viral replication and promotes the nuclear localization of PB2 in human cells, but not avian cells, most likely by facilitating PB2's interaction with importin- $\alpha$ (89). Notably, having PB2 $701 \mathrm{~N}$ can also compensate for the reduced transmissibility imparted by PB2 627E (18). Interestingly, 2009 S-OIVs have neither the PB2 627K nor the $701 \mathrm{~N}$ mutations that are associated with high pathogenicity, although second-site compensatory mutations in PB2 (590S and 591R) have been identified (90).

PB1-F2. PB1-F2 is a small protein of up to 90 amino acids that is encoded from the +1 reading frame of the PB1 gene (4). Various studies have suggested that this protein plays an important role in virulence of primary influenza virus infection and in promoting secondary bacterial infection (7). PB1-F2 has a proapoptotic function in immune cells, proposed to be the result of its association with mitochondrial membranes, where it interacts with voltage-dependent anion channel 1 (VDAC-1) and adenine nucleotide translocator 3 (ANT3) $(4,6)$. At least in vitro, PB1-F2 enhances polymerase activity by binding to the $\mathrm{PB} 1$ polymerase subunit (91). How these molecular functions relate to the pathogenicity of influenza viruses remains the subject of debate.

PB1-F2 was shown to contribute to the pathogenicity of the 1918, 1957, and 1968 pandemic strains, as well H5N1 HPAI viruses $(5,92,93)$. When an S66N mutation was incorporated into the PB1-F2 proteins of $\mathrm{H} 5 \mathrm{~N} 1$ and 1918 viruses, they became attenuated in mice (5). In addition, viruses with an N66S mutation caused increased disease severity, lung titers, and cytokine production in mice (5). Interestingly, 2009 S-OIVs express only a truncated, 11-amino acid PB1-F2 protein, although introduction of PB1F2, either with $66 \mathrm{~N}$ or $66 \mathrm{~S}$, into recombinant $2009 \mathrm{~S}-\mathrm{OIV}$ did not substantially enhance its virulence in mice or ferrets or predispose mice to secondary bacterial infection with Streptococcus pneumoniae (94). PB1-F2 is expressed by nearly all avian influenza viruses, but these proteins tend to become truncated following introduction into humans or pigs (95), which suggests that there is some 
selective advantage for full-length PB1-F2 in the avian host that is lost during adaptation to mammals (92). It may be that PB1-F2 only contributes to the virulence of pandemic strains when the PB1 gene segment is recently derived from an avian reservoir (92). Therefore, the ability of PB1-F2 to promote virulence and pathogenicity is host and strain specific and may be influenced by other factors in the context of the entire viral genome.

NS1. As an IFN antagonist, NS1 forges a multipronged attack against the innate immune response triggered by influenza virus infection $(96,97)$. NS1 prevents activation of transcription factors that induce IFN- $\beta$ by blocking recognition of influenza pathogenassociated molecular patterns (PAMPs) through retinoid-inducible gene-1 (RIG-I) (98). NS1 also interferes with the expression of host ISGs by preventing the proper processing of cellular mRNAs and inhibiting the antiviral activities of ISG products. The NS1 proteins of H5N1 HPAI viruses are associated with the induction of high levels of proinflammatory cytokines in the infected host (99-102). Although this dysregulation of innate immune responses has been linked with fatal H5N1 disease outcomes, it is unknown whether this is directly associated with NS1 function or caused by high levels of replication of these viruses.

Specific residues in NS1 have been linked to influenza virus virulence. H5N1 HPAI viruses that are pathogenic in mice contain a glutamic acid, rather than aspartic acid, at position 92 of NS1 (102). NS1 92E allows viral replication in the presence of IFN in vitro and is linked to pathogenicity in pigs. Likewise, an H5N1 P42S change results in a substantial increase in virulence in the mouse model and reduced levels of IFN- $\alpha / \beta$ production in vitro (103). L103F and I106M mutations in the NS1 of H5N1 viruses, which increase NS1 binding to the cellular pre-mRNA processing protein cleavage and polyadenylation specificity factor (CPSF30), increase in vitro viral replication, presumably by suppressing expression of IFN- $\alpha / \beta$ mRNAs (104). Finally, the carboxyterminal portion of NS1 has also been shown to influence influenza pathogenicity. Pandemic 1918 and H5N1 HPAI viruses contain 4 carboxyterminal amino acids encoding a PDZ ligand domain motif $(105,106)$, which increases virulence when introduced into a mouse-adapted influenza strain (106). The role of the NS1 PDZ ligand domain in pathogenesis remains unknown, but it appears to be independent of NS1 IFN antagonism.

Although it is a satisfactory IFN antagonist, 2009 S-OIV lacks certain NS1 functions, including CPSF30 binding (107). Interestingly, restoring CPSF30 binding activity by substituting the human consensus amino acids, R108K, E125D, and G189D, actually attenuated the virus in mouse and ferret models (107). These data suggest that NS1 biological activity may contribute to pathogenesis in a strain-specific, host species-dependent manner.

$N A$. Optimal influenza virus replication requires a functional balance between HA sialic acid binding affinity and receptordestroying, enzymatic activity of NA (108). This balance can be perturbed by a number of events, such as reassortment, introduction into a novel host, and antiviral therapy. The earliest human isolates of the $1957 \mathrm{H} 2 \mathrm{~N} 2$ pandemic viruses paired an NA with preference for $\alpha 2,3$-linked substrates with an HA that bound well to $\alpha 2,6$-sialic acid receptors. Over the years, this N2 gradually acquired the ability to cleave both $\alpha 2,3$ and $\alpha 2,6$ linkages, adapting to meet the receptor specificity of the HA (109). This likely provided a selective advantage by allowing progeny virions to be released more efficiently from the cell surface.

Antiviral drugs can also influence the adaptation of influenza viruses. Oseltamivir binds to the active site of NA present at the surface of an infected cell (110), preventing it from removing sialic acid residues and causing virus aggregation. In clinical trials with oseltamivir, an NA H274Y resistance mutation in the background of a seasonal H1N1 virus was identified, but deemed clinically unimportant because of its expense on virus fitness (111). Surprisingly, during the influenza season of 2007-2008, oseltamivir-resistant $\mathrm{H} 1 \mathrm{~N} 1$ viruses with the $\mathrm{H} 274 \mathrm{Y}$ mutation began to predominate in the circulating H1N1 population. Recently, second-site mutations in NA that counteract the loss of fitness in the resistant virus have been described (112). Monitoring these resistance mutations and their possible introduction in 2009 S-OIVs will be prudent in upcoming influenza seasons.

Host and environmental factors. Several studies have focused on the complex interplay of cellular factors required for influenza virus replication (113-115). RNAi screens have been especially fruitful in highlighting host genes and pathways that are targeted and/or manipulated by influenza virus (113-115). Multiple factors were identified in several independent screens, which suggests their importance for influenza virus (116). Whether the ability to interact efficiently with particular host factors and to usurp a more diverse set of cellular pathways is associated with virus pathogenicity requires further study. Human polymorphisms affecting cellular proviral or antiviral genes may also play a major role in the outcome of influenza virus infection (117).

In addition, influenza virulence is influenced by the immunological and physiological environment within the host. Preexisting immunity in the older human population, such as was observed in the 2009 S-OIV pandemic, limited infection in these patients, whereas younger children were more susceptible to severe disease. The pathogenic potential of an influenza virus can also be enhanced by coinfection with a bacterial pathogen. The 1918 influenza virus predisposed infected individuals to a secondary bacterial infection, resulting in pneumonia in numerous cases (26).

\section{Conclusions and perspectives}

Although a more virulent S-OIV failed to emerge during the 20092010 influenza season, vigilant surveillance of these strains, as well as H5N1 HPAI viruses, for the features described above is critically important. Some are almost universal markers of pathogenicity - an HA multi-basic cleavage site, a PB2 E627K mutation - whereas others are strain and host specific, such as particular functions of NS1 or PB1-F2. These markers should be monitored in avian species, as well as swine, for early detection of threatening strains. Furthermore, large-scale analysis of viral genomes will continue to identify additional molecular correlates of influenza virulence. These efforts will help to clarify how the constellation of genes composing a particular influenza virus gives rise to a virus with pandemic potential.

\section{Acknowledgments}

Work in A. García-Sastre's lab is supported by NIAID grants R01AI046954, U01AI070469, U19AI083025, U54AI057158, and P01AI058113; by CRIP - an NIAID-funded Center of Excellence for Influenza Research and Surveillance (HHSN266200700010C); and by the W.M. Keck Foundation. D.M. Tscherne is funded by NIAID grant 1F32AI081428.

Address correspondence to: Adolfo García-Sastre, Mount Sinai School of Medicine, Department of Microbiology, Box 1124, One Gustave L. Levy Place, New York, New York 10029, USA. Phone: 212.241.7769; Fax: 212.534.1684; E-mail: adolfo.garcia-sastre@mssm.edu. 
1. Palese P, Shaw ML. Orthomyxoviridae: The viruses and their replication. In: Knipe DM, Howley PM, eds. Fields Virology. 5th ed. Philadelphia, Pennsylvania, USA: Lippincott Williams \& Wilkins; 2007:1647-1689.

2. Webster RG, Bean WJ, Gorman OT, Chambers TM, Kawaoka Y. Evolution and ecology of influenza A viruses. Microbiol Rev. 1992;56(1):152-179.

3. Scheiffele P, Rietveld A, Wilk T, Simons K. Influenza viruses select ordered lipid domains during budding from the plasma membrane.J Biol Chem. 1999;274(4):2038-2044.

4. Chen W, et al. A novel influenza A virus mitochondrial protein that induces cell death. Nat Med. 2001;7(12):1306-1312.

5. Conenello GM, Zamarin D, Perrone LA, Tumpey T, Palese P. A single mutation in the PB1-F2 of H5N1 (HK/97) and 1918 influenza A viruses contributes to increased virulence. PLoS Pathog. 2007; 3(10):1414-1421.

6. Zamarin D, Garcia-Sastre A, Xiao X, Wang R, Palese $\mathrm{P}$. Influenza virus PB1-F2 protein induces cell death through mitochondrial ANT3 and VDAC1. PLoS Pathog. 2005;1(1):e4.

7. McAuley JL, et al. Expression of the 1918 influenza A virus PB1-F2 enhances the pathogenesis of viral and secondary bacterial pneumonia. Cell Host Microbe. 2007;2(4):240-249.

8. Fodor E, Devenish L, Engelhardt OG, Palese P, Brownlee GG, Garcia-Sastre A. Rescue of influenza A virus from recombinant DNA. J Virol. 1999; 73(11):9679-9682.

9. Neumann G, et al. Generation of influenza A viruses entirely from cloned cDNAs. Proc Natl Acad Sci US A. 1999;96(16):9345-9350.

10. Barnard DL. Animal models for the study of influenza pathogenesis and therapy. Antiviral Res. 2009;82(2):A110-122.

11. Svitek N, Rudd PA, Obojes K, Pillet S, von Messling $V$. Severe seasonal influenza in ferrets correlates with reduced interferon and increased IL-6 induction. Virology. 2008;376(1):53-59.

12. Smee DF, et al. Treatment of influenza A (H1N1) virus infections in mice and ferrets with cyanovirin-N. Antiviral Res. 2008;80(3):266-271.

13. Sidwell RW, Smee DF. In vitro and in vivo assay systems for study of influenza virus inhibitors. Antiviral Res. 2000;48(1):1-16.

14. Smith H, Sweet C. Lessons for human influenza from pathogenicity studies with ferrets. Rev Infect Dis. 1988;10(1):56-75.

15. Lowen AC, Mubareka S, Steel J, Palese P. Influenza virus transmission is dependent on relative humidity and temperature. PLoS Pathog. 2007; 3(10):1470-1476.

16. Lowen AC, Steel J, Mubareka S, Carnero E, GarciaSastre A, Palese P. Blocking interhost transmission of influenza virus by vaccination in the guinea pig model. J Virol. 2009;83(7):2803-2818.

17. Lowen AC, Steel J, Mubareka S, Palese P. High temperature (30 degrees $C$ ) blocks aerosol but not contact transmission of influenza virus. $J$ Virol. 2008;82(11):5650-5652.

18. Steel J, Lowen AC, Mubareka S, Palese P. Transmission of influenza virus in a mammalian host is increased by PB2 amino acids $627 \mathrm{~K}$ or $627 \mathrm{E} / 701 \mathrm{~N}$. PLoS Pathog. 2009;5(1):e1000252.

19. Mubareka S, Lowen AC, Steel J, Coates AL, GarcíaSastre A, Palese P. Transmission of influenza virus via aerosols and fomites in the guinea pig model. J Infect Dis. 2009;199(6):858-865.

20. van der Laan JW, Herberts C, Lambkin-Williams R, Boyers A, Mann AJ, Oxford J. Animal models in influenza vaccine testing. Expert Rev Vaccines. 2008;7(6):783-793.

21. Baas T, et al. Integrated molecular signature of disease: analysis of influenza virus-infected macaques through functional genomics and proteomics.
J Virol. 2006;80(21):10813-10828.

22. Glezen WP. Emerging infections: pandemic influenza. Epidemiol Rev. 1996;18(1):64-76.

23. Taubenberger JK, Morens DM. Pandemic influenza--including a risk assessment of H5N1. Rev Sci Tech. 2009;28(1):187-202.

24. Johnson NP, Mueller J. Updating the accounts: global mortality of the 1918-1920 "Spanish" influenza pandemic. Bull Hist Med. 2002;76(1):105-115.

25. Taubenberger JK, Morens DM. 1918 Influenza: the mother of all pandemics. Emerg Infect Dis. 2006;12(1):15-22.

26. Morens DM, Taubenberger JK, Fauci AS. Predominant role of bacterial pneumonia as a cause of death in pandemic influenza: implications for pandemic influenza preparedness. J Infect Dis. 2008; 198(7):962-970.

27. Basler CF, et al. Sequence of the 1918 pandemic influenza virus nonstructural gene (NS) segment and characterization of recombinant viruses bearing the 1918 NS genes. Proc Natl Acad Sci US A. 2001; 98(5):2746-2751.

28. Taubenberger JK, Reid AH, Krafft AE, Bijwaard KE, Fanning TG. Initial genetic characterization of the 1918 "Spanish" influenza virus. Science. 1997; 275(5307):1793-1796.

29. Tumpey TM, et al. Characterization of the reconstructed 1918 Spanish influenza pandemic virus. Science. 2005;310(5745):77-80.

30. Tumpey TM, et al. A two-amino acid change in the hemagglutinin of the 1918 influenza virus abolishes transmission. Science. 2007;315(5812):655-659.

31. Kobasa D, et al. Aberrant innate immune response in lethal infection of macaques with the 1918 influenza virus. Nature. 2007;445(7125):319-323.

32. Weingartl HM, et al. Experimental infection of pigs with the human 1918 pandemic influenza virus. J Virol. 2009;83(9):4287-4296.

33. Van Hoeven N, et al. Human HA and polymerase subunit PB2 proteins confer transmission of an avian influenza virus through the air. Proc Natl Acad Sci U S A. 2009;106(9):3366-3371.

34. Kash JC, et al. Genomic analysis of increased host immune and cell death responses induced by 1918 influenza virus. Nature. 2006;443(7111):578-581.

35. Scholtissek C, Rohde W, Von Hoyningen V, Rott R. On the origin of the human influenza virus subtypes H2N2 and H3N2. Virology. 1978;87(1):13-20.

36. Kawaoka Y, Krauss S, Webster RG. Avian-tohuman transmission of the PB1 gene of influenza A viruses in the 1957 and 1968 pandemics. J Virol. 1989;63(11):4603-4608.

37. Kilbourne ED. Influenza pandemics of the 20th century. Emerg Infect Dis. 2006;12(1):9-14.

38. Nakajima K, Desselberger U, Palese P. Recent human influenza A (H1N1) viruses are closely related genetically to strains isolated in 1950. Nature. 1978;274(5669):334-339.

39. Rambaut A, Pybus OG, Nelson MI, Viboud C, Taubenberger JK, Holmes EC. The genomic and epidemiological dynamics of human influenza A virus. Nature. 2008;453(7195):615-619.

40. Gregory V, et al. Emergence of influenza A H1N2 reassortant viruses in the human population during 2001. Virology. 2002;300(1):1-7.

41. CDC. Update: influenza activity--United States and worldwide, May 22-September 3, 2005, and 2005-06 season vaccination recommendations [published correction appears in MMWR Morb Mortal Wkly Rep. 2005;54(37):935]. MMWR Morb Mortal Wkly Rep. 2005;54(36):899-902.

42. Fraser $C$, et al. Pandemic potential of a strain of influenza A (H1N1): early findings. Science. 2009;324(5934):1557-1561.

43. Global Alert and Response, Pandemic (H1N1) 2009. WHO Web site. http://www.who.int/csr/ disease/swineflu/en/. Accessed November 8, 2010. 44. Dunham EJ, et al. Different evolutionary tra- jectories of European avian-like and classical swine H1N1 influenza A viruses. J Virol. 2009; 83(11):5485-5494.

45. Garten RJ, et al. Antigenic and genetic characteristics of swine-origin 2009 A(H1N1) influenza viruses circulating in humans. Science. 2009; 325(5937):197-201.

46. Smith GJ, et al. Origins and evolutionary genomics of the 2009 swine-origin H1N1 influenza A epidemic. Nature. 2009;459(7250):1122-1125.

47. Shinde V, et al. Triple-reassortant swine influenza A (H1) in humans in the United States, 2005-2009. NEngl J Med. 2009;360(25):2616-2625.

48. Dawood FS, et al. Emergence of a novel swine-origin influenza A (H1N1) virus in humans. $N$ Engl J Med. 2009;360(25):2605-2615.

49. Maines TR, et al. Transmission and pathogenesis of swine-origin $2009 \mathrm{~A}(\mathrm{H} 1 \mathrm{~N} 1)$ influenza viruses in ferrets and mice. Science. 2009;325(5939):484-487.

50. Munster VJ, et al. Pathogenesis and transmission of swine-origin $2009 \mathrm{~A}(\mathrm{H} 1 \mathrm{~N} 1)$ influenza virus in ferrets. Science. 2009;325(5939):481-483.

51. Manicassamy B, et al. Protection of mice against lethal challenge with $2009 \mathrm{H} 1 \mathrm{~N} 1$ influenza A virus by 1918 -like and classical swine H1N1 based vaccines. PLoS Pathog. 2010;6(1):e1000745.

52. Xu R, Ekiert DC, Krause JC, Hai R, Crowe JE Jr, Wilson IA. Structural basis of preexisting immunity to the 2009 H1N1 pandemic influenza virus. Science. 2010;328(5976):357-360.

53. Wei CJ, et al. Cross-neutralization of 1918 and 2009 influenza viruses: role of glycans in viral evolution and vaccine design. Sci Transl Med. 2010;2(24):24ra21.

54. Itoh $\mathrm{Y}$, et al. In vitro and in vivo characterization of new swine-origin $\mathrm{H} 1 \mathrm{~N} 1$ influenza viruses. Nature. 2009;460(7258):1021-1025.

55. Perez DR, et al. Fitness of pandemic H1N1 and seasonal influenza A viruses during co-infection: evidence of competitive advantage of pandemic H1N1 influenza versus seasonal influenza. PLoS Curr. 2009;1:RRN1011.

56. Subbarao K, et al. Characterization of an avian influenza A (H5N1) virus isolated from a child with a fatal respiratory illness. Science. 1998; 279(5349):393-396.

57. Claas EC, et al. Human influenza A H5N1 virus related to a highly pathogenic avian influenza virus. Lancet. 1998;351(9101):472-477.

58. Abdel-Ghafar AN, et al. Update on avian influenza A (H5N1) virus infection in humans. $N$ Engl J Med. 2008;358(3):261-273.

59. de Jong MD, et al. Fatal outcome of human influenza A (H5N1) is associated with high viral load and hypercytokinemia. Nat Med. 2006;12(10):1203-1207.

60. Wang $\mathrm{H}$, et al. Probable limited person-to-person transmission of highly pathogenic avian influenza A (H5N1) virus in China. Lancet. 2008; 371(9622):1427-1434.

61. Cumulative number of confirmed cases of avian influenza $\mathrm{A} /(\mathrm{H} 5 \mathrm{~N} 1)$ reported to the WHO. WHO Web site. http://www.who.int/csr/disease/avian_ influenza/country/en/. Accessed November 8, 2010.

62. Yang Y, Halloran ME, Sugimoto JD, Longini IM Jr. Detecting human-to-human transmission of avian influenza A (H5N1). Emerg Infect Dis. 2007;13(9):1348-1353.

63. Ito $\mathrm{T}$, et al. Molecular basis for the generation in pigs of influenza A viruses with pandemic potential. J Virol. 1998;72(9):7367-7373.

64. Rogers GN, Paulson JC. Receptor determinants of human and animal influenza virus isolates: differences in receptor specificity of the $\mathrm{H} 3 \mathrm{hem}$ agglutinin based on species of origin. Virology. 1983;127(2):361-373.

65. Matrosovich $\mathrm{M}$, et al. Early alterations of the receptor-binding properties of $\mathrm{H} 1, \mathrm{H} 2$, and $\mathrm{H} 3$ avian influenza virus hemagglutinins after their introduc- 
tion into mammals. J Virol. 2000;74(18):8502-8512. 66. Connor RJ, Kawaoka Y, Webster RG, Paulson JC. Receptor specificity in human, avian, and equine H2 and H3 influenza virus isolates. Virology. 1994; 205(1):17-23.

67. Stevens J, Corper AL, Basler CF, Taubenberger JK, Palese P, Wilson IA. Structure of the uncleaved human H1 hemagglutinin from the extinct 1918 influenza virus. Science. 2004;303(5665):1866-1870.

68. Glaser $\mathrm{L}$, et al. A single amino acid substitution in 1918 influenza virus hemagglutinin changes receptor binding specificity. JVirol. 2005;79(17):11533-11536.

69. Auewarakul P, et al. An avian influenza H5N1 virus that binds to a human-type receptor. J Virol. 2007;81(18):9950-9955.

70. Gambaryan A, Tuzikov A, Pazynina G, Bovin N, Balish A, Klimov A. Evolution of the receptor binding phenotype of influenza A (H5) viruses. Virology. 2006;344(2):432-438

71. Stevens J, Blixt O, Tumpey TM, Taubenberger JK Paulson JC, Wilson IA. Structure and receptor specificity of the hemagglutinin from an $\mathrm{H} 5 \mathrm{~N} 1$ influenza virus. Science. 2006;312(5772):404-410.

72. Yamada S, et al. Haemagglutinin mutations responsible for the binding of $\mathrm{H} 5 \mathrm{~N} 1$ influenza A viruses to human-type receptors. Nature. 2006 444(7117):378-382.

73. Yang $Z Y$, et al. Immunization by avian $\mathrm{H} 5$ influenza hemagglutinin mutants with altered receptor binding specificity. Science. 2007;317(5839):825-828.

74. Shinya K, Ebina M, Yamada S, Ono M, Kasai N, Kawaoka Y. Avian flu: influenza virus receptors in the human airway. Nature. 2006;440(7083):435-436.

75. van Riel D, et al. H5N1 Virus Attachment to Lower Respiratory Tract. Science. 2006;312(5772):399.

76. Garten W, Klenk HD. Understanding influenza virus pathogenicity. Trends Microbiol. 1999;7(3):99-100.

77. Horimoto T, Kawaoka Y. Pandemic threat posed by avian influenza A viruses. Clin Microbiol Rev. 2001; 14(1):129-149.

78. Steinhauer DA. Role of hemagglutinin cleavage for the pathogenicity of influenza virus. Virology. 1999; 258(1):1-20.

79. Kawaoka Y, Webster RG. Sequence requirements for cleavage activation of influenza virus hemagglutinin expressed in mammalian cells. Proc Natl Acad Sci U S A. 1988;85(2):324-328.

80. Hatta M, Gao P, Halfmann P, Kawaoka Y. Molecular basis for high virulence of Hong Kong H5N1 influenza A viruses. Science. 2001;293(5536):1840-1842.

81. Hatta M, et al. Growth of H5N1 influenza A viruses in the upper respiratory tracts of mice. PLoS Pathog. 2007;3(10):1374-1379.

82. Mase M, Tanimura N, Imada T, Okamatsu M, Tsukamoto K, Yamaguchi S. Recent H5N1 avian influenza A virus increases rapidly in virulence to mice after a single passage in mice. J Gen Virol. 2006;87(pt 12):3655-3659.

83. Smith GJ, et al. Emergence and predominance of an H5N1 influenza variant in China. Proc Natl Acad Sci US A. 2006;103(45):16936-16941.

84. Puthavathana $P$, et al. Molecular characterization of the complete genome of human influenza H5N1 virus isolates from Thailand. J Gen Virol. 2005; 86(pt 2):423-433

85. Massin P, van der Werf S, Naffakh N. Residue 627 of PB2 is a determinant of cold sensitivity in RNA replication of avian influenza viruses. $J$ Virol. 2001;75(11):5398-5404.

86. Rameix-Welti MA, Tomoiu A, Dos Santos Afonso E, van der Werf S, Naffakh N. Avian Influenza A virus polymerase association with nucleoprotein, but not polymerase assembly, is impaired in human cells during the course of infection. J Virol. 2009; 83(3):1320-1331.

87. Mehle A, Doudna JA. An inhibitory activity in human cells restricts the function of an avianlike influenza virus polymerase. Cell Host Microbe. 2008;4(2):111-122.

88. Moncorge O, Mura M, Barclay WS. Evidence for avian and human host cell factors that affect the activity of influenza virus polymerase. J Virol. 2010; 84(19):9978-9986.

89. Gabriel G, Herwig A, Klenk HD. Interaction of polymerase subunit PB2 and NP with importin alpha1 is a determinant of host range of influenza A virus. PLoS Pathog. 2008;4(2):e11.

90. Mehle A, Doudna JA. Adaptive strategies of the influenza virus polymerase for replication in humans. Proc Natl Acad Sci U S A. 2009;106(50):21312-21316.

91. Mazur I, Anhlan D, Mitzner D, Wixler L, Schubert $\mathrm{U}$, Ludwig S. The proapoptotic influenza A virus protein PB1-F2 regulates viral polymerase activity by interaction with the PB1 protein. Cell Microbiol. 2008;10(5):1140-1152.

92. McAuley JL, Chipuk JE, Boyd KL, Van De Velde N, Green DR, McCullers JA. PB1-F2 proteins from $\mathrm{H} 5 \mathrm{~N} 1$ and 20 century pandemic influenza viruses cause immunopathology. PLoS Pathog. 2010; 6(7):e1001014.

93. Zamarin D, Ortigoza MB, Palese P. Influenza A virus $\mathrm{PB} 1-\mathrm{F} 2$ protein contributes to viral pathogenesis in mice. J Virol. 2006;80(16):7976-7983

94. Hai R, et al. PB1-F2 expression by the 2009 pandemic $\mathrm{H} 1 \mathrm{~N} 1$ influenza virus has minimal impact on virulence in animal models. J Virol. 2009; 84(9):4442-4450.

95. Zell R, Krumbholz A, Eitner A, Krieg R, Halbhuber KJ, Wutzler P. Prevalence of PB1-F2 of influenza A viruses. J Gen Virol. 2007;88(pt 2):536-546.

96. Garcia-Sastre A. Inhibition of interferon-mediated antiviral responses by influenza A viruses and other negative-strand RNA viruses. Virology. 2001; 279(2):375-384.

97. Garcia-Sastre A, et al. Influenza A virus lacking the NS1 gene replicates in interferon-deficient systems. Virology. 1998;252(2):324-330.

98. Hale BG, Randall RE, Ortin J, Jackson D. The multifunctional NS1 protein of influenza A viruses. J Gen Virol. 2008;89(pt 10):2359-2376.

99. To KF, et al. Pathology of fatal human infection associated with avian influenza A H5N1 virus. JMed Virol. 2001;63(3):242-246.

100.Peiris JS, et al. Re-emergence of fatal human influenza A subtype H5N1 disease. Lancet. 2004; 363(9409):617-619.
101. Cheung CY, et al. Induction of proinflammatory cytokines in human macrophages by influenza A (H5N1) viruses: a mechanism for the unusual severity of human disease? Lancet. 2002; 360(9348):1831-1837.

102.Seo SH, Hoffmann E, Webster RG. Lethal H5N1 influenza viruses escape host anti-viral cytokine responses. Nat Med. 2002;8(9):950-954.

103.Jiao P, et al. A single-amino-acid substitution in the NS1 protein changes the pathogenicity of $\mathrm{H} 5 \mathrm{~N} 1$ avian influenza viruses in mice. J Virol. 2008;82(3):1146-1154.

104. Twu KY, Kuo RL, Marklund J, Krug RM. The H5N1 influenza virus NS genes selected after 1998 enhance virus replication in mammalian cells. $J$ Virol. 2007;81(15):8112-8121.

105. Obenauer JC, et al. Large-scale sequence analysis of avian influenza isolates. Science. 2006; 311(5767):1576-1580.

106.Jackson D, Hossain MJ, Hickman D, Perez DR, Lamb RA. A new influenza virus virulence determinant: the NS1 protein four C-terminal residues modulate pathogenicity. Proc Natl Acad Sci U S A. 2008;105(11):4381-4386

107. Hale BG, et al. Inefficient control of host gene expression by the 2009 pandemic H1N1 influenza A virus NS1 protein. J Virol. 2010;84(14):6909-6922.

108. Wagner R, Matrosovich M, Klenk HD. Functional balance between haemagglutinin and neuraminidase in influenza virus infections. Rev Med Virol. 2002;12(3):159-166.

109. Baum LG, Paulson JC. The N2 neuraminidase of human influenza virus has acquired a substrate specificity complementary to the hemagglutinin receptor specificity. Virology. 1991;180(1):10-15.

110. Moscona A. Oseltamivir resistance--disabling our influenza defenses. N Engl J Med. 2005; 353(25):2633-2636

111. Ives JA, et al. The H274Y mutation in the influenza A/H1N1 neuraminidase active site following oseltamivir phosphate treatment leave virus severely compromised both in vitro and in vivo. Antiviral Res. 2002;55(2):307-317.

112.Bloom JD, Gong LI, Baltimore D. Permissive secondary mutations enable the evolution of influenza oseltamivir resistance. Science. 2010; 328(5983):1272-1275.

113. Hao L, et al. Drosophila RNAi screen identifies host genes important for influenza virus replication. Nature. 2008;454(7206):890-893.

114. Brass AL, et al. The IFITM proteins mediate cellular resistance to influenza $\mathrm{A} \mathrm{H} 1 \mathrm{~N} 1$ virus, West Nile virus, and dengue virus. Cell. 2009;139(7):1243-1254.

115.Konig R, et al. Human host factors required for influenza virus replication. Nature. 2010; 463(7282):813-817.

116. Watanabe T, Watanabe S, Kawaoka Y. Cellular networks involved in the influenza virus life cycle. Cell Host Microbe. 2010;7(6):427-439.

117. Albright FS, Orlando P, Pavia AT, Jackson GG, Cannon Albright LA. Evidence for a heritable predisposition to death due to influenza. J Infect Dis. 2008;197(1):18-24. 